\title{
diffusion-Funiclameritals.org
}

The Open-Access Journal for the Basic Principles of Diffusion Theory, Experiment and Application

\section{Molecular Dynamics Simulation Study of the Concentration Dependence of the Self-Diffusivity of Methanol in NaX Zeolite}

\author{
T. Nanok ${ }^{l}$, O. Sangsawang ${ }^{l}$, S. Vasenkov ${ }^{2}$, F. J. Keil ${ }^{3}, \underline{\text { S. Fritzsche }}^{l}$ \\ ${ }^{1}$ Universität Leipzig, Fakultät für Physik und Geowissenschaften, Postfach 100920, \\ 04009 Leipzig, Germany, E-Mail: siegfried.fritzsche@uni-leipzig.de \\ ${ }^{2}$ University of Florida, Department of Chemical Engineering, Gainesville, Florida, USA \\ ${ }^{3}$ Department of Chemical Engineering, Hamburg University of Technology, D-21073 \\ Hamburg, Germany
}

\section{Introduction}

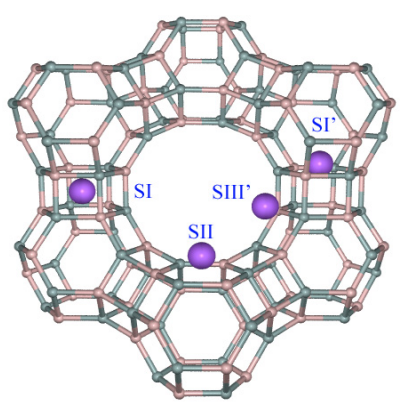

Fig. 1: Illustration of the $\mathrm{Na}^{+}$ ions located at different sites in the $\mathrm{NaX}$ crystallographic structure.
Nanoporous aluminosilicate zeolites containing extraframework alkali cations have become increasingly important in chemical science and technology due to their potential applications in many processes including catalysis, storage and separation. For all applications, the diffusion of guest molecules in their confined geometry plays a crucial role because it can limit the speed and efficiency of all processes. In general, the diffusivity of molecules decreases with increasing loading because of mutual hindrance. In some cases, especially in the cation-exchanged zeolites, it, however, passes through a maximum at a particular loading [1,2]. The explanation of such maximum in zeolites with various compositions and structures can be different. In this contribution, a microscopic interpretation of the concentration dependence of the self-diffusivity of methanol in $\mathrm{NaX}$ zeolite will be provided by using-Molecular Dynamics (MD) simulations.

\section{Models and Simulations}

The MD simulations were carried out on one periodic unit cell of the $\mathrm{NaX}$ zeolite having the composition of $\mathrm{Na}_{96} \mathrm{Si}_{96} \mathrm{Al}_{96} \mathrm{O}_{384}$. The 64 extra framework $\mathrm{Na}^{+}$ions were distributed to the SI' and SII sites (Fig. 1) as established by the previous powder neutron diffraction study [3]. The remaining $32 \mathrm{Na}^{+}$ions were distributed to the SIII' sites having equally two $\mathrm{Na}^{+}$ions per window. Methanol molecules were randomly distributed in the supercages. The temperature of this system was increased to achieve a more uniform distribution over the zeolite lattice. The resulting configuration was thermalized at the required temperature for at least $1 \mathrm{~ns}$ followed by the production run of $5 \mathrm{~ns}$.

(C) 2009, S. Fritzsche

diffusion-fundamentals.org 11 (2009) 33, pp 1-2 


\section{Results and Discussion}

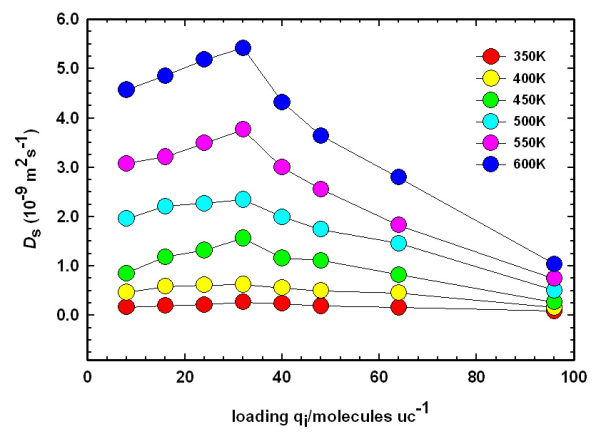

Fig. 2: Self-diffusion coefficients of methanol in $\mathrm{NaX}$ at different loadings and temperatures.
The results of the self-diffusion coefficients of methanol in NaX zeolites by MD simulations are shown in Fig. 2. For each temperature, the self-diffusivity continuously increases with increasing loading and reaches the maximum value at the loading of 32 molecules per unit cell. At higher loadings it gradually decreases with increasing loading until the saturation limit is reached at 96 molecules per unit cell. These results are in a qualitative agreement with the experimental concentration dependence of the self-diffusivity [1]. An evaluated first shell coordination number of $\mathrm{Na}^{+}$ions as a function of the loading shows that the SIII' is the most preferable site for methanol adsorption. If the loading increases up to 32 molecules per unit cell, methanol molecules are adsorbed on almost every $\mathrm{Na}^{+}$ ion located at the SIII'. The collective effects from the mutual repulsion between methanol molecules competing for this site can, therefore, enhance the rate of intercage diffusion. An analysis of the mean square displacement of $\mathrm{Na}^{+}$ions as a function of time reveals that the $\mathrm{Na}^{+}$-methanol collective motion could not be ruled out. The $\mathrm{Na}^{+}$ions located at the SIII' sites can migrate out of their initial positions upon the adsorption of methanol. This migration is, however, considered to be very slow as compared to the diffusion of methanol molecules. Thus, the preferable adsorption of two adjacent methanol molecules at the SIII' sites can possibly block the 12-membered ring (MR) window, resulting in a slow down of the intercage diffusion at loadings higher than 32 molecules per unit cell.

\section{Conclusion}

The analyses of the MD trajectories can provide microscopic information about the concentration dependence of the self-diffusivity of methanol in $\mathrm{NaX}$ zeolite. In addition to the collective effects from the mutual repulsion between methanol molecules preferably adsorbing at the 12-MR window, the collective cation-methanol motion could also play a role in enhancing the self-diffusion. The blocking of the 12-MR window at high loadings by two methanol molecules competing for each of the SIII' sites leads to a decrease in the intercage diffusivity.

\section{References}

[1] S. Brandani, D. M. Ruthven, J. Kärger, Zeolites 15 (1995) 494-495.

[2] D. F. Plant, G. Maurin, R.G. Bell, J. Phys. Chem. B 110 (2006) 15926-15931.

[3] G. Vitale, C.F. Mellot, L.M. Bull, A.K. Cheetham, J. Phys. Chem. B 101 (1997) 4559-4564. 